\title{
Signal improvement in multiphoton microscopy by reflection with simple mirrors near the sample
}

\author{
Markus Rehberg \\ Fritz Krombach \\ Ulrich Pohl \\ Steffen Dietzel \\ Ludwig-Maximilians-Universität München \\ Walter-Brendel-Zentrum für Experimentelle Medizin \\ Marchioninistrasse 27 \\ München, D-81377 Germany
}

\begin{abstract}
In conventional fluorescence or confocal microscopy, emitted light is generated not only in the focal plane but also above and below. The situation is different in multiphoton-induced fluorescence and multiphoton-induced higher harmonic generation. Here, restriction of signal generation to a single focal point permits that all emitted photons can contribute to image formation if collected, regardless of their path through the specimen. Often, the intensity of the emitted light is rather low in biological specimens. We present a method to significantly increase the fraction of photons collected by an epi (backward) detector by placing a simple mirror, an aluminum-coated coverslip, directly under the sample. Samples investigated include fluorescent test slides, collagen gels, and thin-layered, intact mouse skeletal muscles. Quantitative analysis revealed an intensity increase of second- and third-harmonic generated signal in skeletal muscle of nine- and sevenfold respectively, and of fluorescent signal in test slides of up to twofold. Our approach thus allows significant signal improvement also for situations were a forward detection is impossible, e.g., due to the anatomy of animals in intravital microscopy. (C) 2010 Society of Photo-Optical Instrumentation Engineers. [DOI: 10.1117/1.3374337]
\end{abstract}

Keywords: Multiphoton microscopy; second-harmonic generation; third-harmonic generation; signal enhancement; mirror.

Paper 09511R received Nov. 18, 2009; revised manuscript received Feb. 8, 2010 accepted for publication Feb. 12, 2010; published online Apr. 20, 2010.

\section{Introduction}

Achievable resolution in biological light microscopy is limited not only by diffraction but also by photon statistics and thus signal intensity. ${ }^{1}$ In fluorescence microscopy, signal intensity and thus the signal-to-noise ratio (SNR) are restrained by excitation power, as increased excitation also leads to increased photobleaching and phototoxicity. While phototoxicity is generally reduced in multiphoton-induced fluorescence due to the smaller excitation volume, ${ }^{2}$ the signal-to-noise-ratio often still presents a problem-in particular, in thick samples, where scattering causes a significant reduction of collected photons. The fluorescence induced in a microscopic sample distributes equally in all spatial directions. Thus, most generated photons are not collected, as the detection angle typically is limited to a fraction of the surrounding space. Even an oil immersion lens with a numerical aperture of 1.4 and thus an angular aperture of $134 \mathrm{deg}$ covers only $30 \%$ of the volume around the focal point and collects only this fraction of the generated fluorescence (calculated from the surface formula for a spherical cap). Collecting emitted photons from additional spatial angles is an obvious desire, and indeed some advanced techniques such as four-Pi-microscopy ${ }^{3,4}$ and Thetamicroscopy do so, ${ }^{5}$ albeit as a side effect in efforts to increase resolution. However, these techniques require specialized op-

Address all correspondence to: Steffen Dietzel, Ludwing-MaximiliansUniversität München, Walter-Brendel-Zentrum für Experimentelle Medizin, Marchioninistrasse 27, München, D-81377 Germany. Tel: 49-89-218076509 Fax: 49-89-21809976509; E-mail: dietzel@Imu.de tical setups that impose spatial restrictions that preclude applications with large samples such as intravital microscopy.

The straightforward approach of reflecting a part of the emitted photons back toward the collecting lens does not work if reflected photons stem from out-of-focus regions. In conventional epifluorescence microscopy, this would reduce the signal-to-noise ratio, while in confocal laser scanning microscopy, the reflected photons would not pass the confocal pinhole. These limitations do not apply to multiphotoninduced signals, however. As opposed to other approaches, in this type of laser scanning microscopy, at any moment, a signal is induced only at a single point of the specimen. Thus, any collected photon will increase the signal-to-noise ratio, regardless of its path through the microscopic sample.

While induced fluorescence distributes equally in all directions, this is not the case for signals from higher harmonic generation ( $\mathrm{HHG})$, an approach that allows high-resolution microscopy without energy deposit in the sample, thus avoiding bleaching and phototoxicity issues (for reviews, see Refs. 6 and 7). Second-harmonic generation (SHG) and thirdharmonic generation (THG) signals are induced parallel to the direction of the incoming laser beam. ${ }^{8,9}$ Thus, HHG signals detected in a forward direction were described to be about several times stronger than signals in the backward direction. ${ }^{10}$ Accordingly, forward detection of SHG and/or THG was applied in most studies of transparent samples (e.g., Refs. 11-13), requiring forward detectors and relaying optical

1083-3668/2010/15(2)/026017/7/\$25.00 @ $2010 \mathrm{SPIE}$ 
hardware that are not standard parts on multiphoton microscopes. Furthermore, in some biological microscopic applications such as intravital microscopy, detection of forward signal is impossible even in transparent samples due to spatial restrictions caused by the anatomy of the animal, preventing the correct positioning of objective and condenser at the same time.

A mirror directly behind the sample, reflecting forwardgenerated photons back toward the objective, theoretically can circumvent these problems. While this appears to be a simple idea, we found only one previous publication mentioning such a setup: ${ }^{14}$ lithium triborate crystals were subjected to SHG, but no characterization of mirrored signal or comparison with conventional detection was given. In another study, a dichroic mirror was inserted behind an oil immersion condenser to reflect two-photon-induced fluorescence, ${ }^{15}$ thus imposing the same spatial restrictions on the sample as forward detection. For a uniformly fluorescent sample, the authors found an intensity increase between 24 and $58 \%$, depending on excitation wavelength and objective used. Higher harmonic generation was not investigated. Both approaches were not applied to biological specimens. A third publication introduced a parabolic, reflective housing around the sample to allow "total emission detection" for two-photon-induced fluorescence with the help of an additional detector in the forward direction. ${ }^{16}$ While the theoretical efficiency of this elegant approach cannot be surpassed, the spatial restrictions it imposes on sample geometry severely limit its usefulness for large samples, as for example, in intravital microscopy. We are not aware of any follow-up publications applying these three setups.

In the present study, we measured whether detection of signals from multiphoton-induced fluorescence and from HHG in a backward direction can be improved by placing a cheap, disposable mirror under the sample, thus increasing the spatial angle from which emitted photons are collected. Such mirrors, conventional coverslips coated with aluminum, have been used previously in reflected-light oblique transillumination (RLOT) microscopy. ${ }^{17}$ Consistent with the spatial distribution of generated signals in the sample, we found a stronger increase in mirror-augmented HHG signals than in fluorescence signals.

\section{Material and Methods}

\subsection{Mirrors}

Mirrors consisted of a round standard cover glass (thickness 0.19 to $0.22 \mathrm{~mm}$, diameter $11.8 \mathrm{~mm}$; E. Hefele Medizintechnik, Munich, Germany), coated with aluminum vapor (performed by NiWe Decor GmbH, Kaufbeuren, Germany). This technique of mass coating is used, e.g., in manufacturing costume jewelry and is thus a low-cost procedure, in the range of 20 Euro-cents or less per mirror.

\subsection{Microscope Setup}

Microscopic observations were performed on a TriMScope (LaVision Biotec, Bielefeld, Germany) built around an Olympus BX 51 microscope (Olympus, Hamburg, Germany) and equipped with a tunable Ultra II Titan:Sapphire laser (Coherent, Dieburg, Germany) and an optical parametric oscillator (OPO; APE, Berlin, Germany) that is pumped by the Ti:Sa. The beam multiplexer of the TriMScope was not used in the current study. While the Ti:Sa excitation beam consists of two parts that are polarized orthogonal to each other, the OPOgenerated beam is polarized in one direction only.

The OPO was used to generate $1270 \mathrm{~nm}$ light $(700 \mathrm{~mW})$. The unattenuated intensity at the sample (i.e., after the objective) was measured with 250 to $300 \mathrm{~mW}$. An Olympus XLUMPlanFl $20 \times / 0.95-\mathrm{W}$ objective was used (working distance $2 \mathrm{~mm}$ ). The following detection channels were used for backward (epi) detection: blue (417 to $477 \mathrm{~nm}$ ), orange $(550$ to $600 \mathrm{~nm})$, and red $(604$ to $644 \mathrm{~nm})$. An additional blue channel was available in the forward direction. 700-nm short-pass filters blocked out excitation light. Light collection in the forward direction was performed by an Olympus WIUCD condenser, NA 0.8. If not required for forward detection, the condenser was removed to avoid reflection of light at its surfaces. Photomultiplier tubes (PMTs) were Hamamatsu H6780-01 for the blue channels, H6780-20 for the others.

\subsection{Fluorescent Test Slides}

Fluorescent plastic slides from Chroma Technology (Rockingham, Vermont) were used. These slides have a high batch-tobatch variability of their excitation and emission behavior. Also, the excitation spectrum is not continuous but shows alternating maxima and minima. Thus, a suitable excitation wavelength must be determined for each slide. We used the following settings: blue slide, 800 -nm excitation, detection in blue channel; red slide, $1040 \mathrm{~nm}$, detection in orange channel. Mirrors and uncoated control coverslips were attached to the underside of the slide with adhesive tape. 3-D stacks were recorded starting above and stopping below the slide. Actual thickness was determined with a sliding caliper to be $1.8 \mathrm{~mm}$. The optical thickness-i.e., the distance the stage had to be moved until the focus plane reached the bottom of the slidewas about $1.45 \mathrm{~mm}$, due to refraction index mismatch between the immersion water and the plastic slide. ${ }^{18}$ To obtain the true difference from the surface of the slide to the focal plane, depths have thus to be multiplied by $1.8 / 1.45=1.24$. Images were recorded with $500 \mu \mathrm{m}, 512$ pixels (px), $800 \mathrm{~Hz}$, with $20-$ or $50-\mu \mathrm{m} z$ distance. The measured average intensity of each section was calculated with ImageJ $1.41 \mathrm{o}$, a public-domain software (W.S. Rasband, U.S. National Institutes of Health, Bethesda, Maryland; http://rsbweb.nih.gov/ $\mathrm{ij} /)$.

\subsection{Collagen Gel}

Collagen gel (PureCol, Advanced BioMatrix, San Diego, California) preparation was done according to Reichardt et al. ${ }^{19}$ with minor modifications. A semicircle-shaped mirror and a respective uncoated control coverslip were placed side by side on a microscopic slide, and the gel was cast on top, using a rubber o-ring as barrier. The optical thickness (see earlier) of the gel varied and was 1300 to $1450 \mu \mathrm{m}$. SHG recording was performed in the blue channels with excitation $\lambda=850 \mathrm{~nm}, 75-\mu \mathrm{m}(512 \mathrm{px})$ image width, and $20-\mu \mathrm{m}$ distances between slices. We observed that too high laser intensities (20\% of maximal power) led to plasma formation in the gel, while too low intensities $(<5 \%)$ did not generate a reasonable signal-to-noise ratio in the backward detector. We thus operated the laser at $10 \%$ of maximal power. Image analysis was performed in ImageJ. Each optical section was 
first median filtered for noise reduction (radius 1). The threshold on each section was increased iteratively until $\leqslant 5 \%$ of the pixels were left. This part of the macro was kindly provided by Jerome Mutterer, Strasbourg, France. For the pixels above threshold, the average intensity was calculated as a measure for SHG signal intensity. The average background signal was calculated from the $\leqslant 5 \%$ of the pixels with the darkest gray values. Arrangement of all presented figures was performed in Adobe Photoshop CS2.

\subsection{Mouse Cremaster Preparation}

Male C57BL/6 mice (age 10 to 12 weeks) were purchased from Charles River (Sulzfeld, Germany). Animals were housed under conventional conditions with free access to food and water. Tissue extraction was performed according to German legislation for the protection of animals. The animals were euthanized by an intraperitoneal pentobarbital overdose (Narcoren, Merial, Germany). Subsequently, the right cremaster muscle was exposed through a ventral incision of the scrotum. The muscle was opened ventrally, and epididymis and testicle were detached. The cremaster was explanted, placed on a standard microscopic glass slide, with or without mirror underneath, and covered with $0.9 \% \mathrm{NaCl}$. On two sides, the edges of the muscle were weighed down with coverslips to avoid floating of the tissue. These muscle preparations have a thickness of about $200 \mu \mathrm{m}$. $0.9 \% \mathrm{NaCl}$ was used for immersion. Observation was performed in the central part, without coverslips between muscle and objective. Imaging was either with $1270 \mathrm{~nm}$ [at $80 \%$ total OPO power (i.e., about $200 \mathrm{~mW}$ behind the objective) with squared images, $1600 \mathrm{px}$, $400 \mu \mathrm{m}$ ] or with $850 \mathrm{~nm}$ ( $2 \%$ TiSa Power, squared images, $512 \mathrm{px}, 400 \mu \mathrm{m})$.

\section{Results}

\subsection{Fluorescence}

To investigate whether two-photon-induced fluorescence signal intensity can be improved by an underlying mirror, we used two fluorescent test slides with different spectral properties. Z-scans were performed from top to bottom of the slides, with an uncoated control coverslip or a mirror (coverslip coated with aluminum) underneath. Intensity distributions showed the highest fluorescence near the surface of the slide (Fig. 1). Intensity dropped to less than half in the first $0.5 \mathrm{~mm}$. Comparison with fluorescence signal intensity detected in the forward direction (data not shown) showed that this was due to absorption and/or scattering of excitation light and thus due to reduced occurrence of the two-photon effect.

Comparison of fluorescence intensities obtained with and without mirrors revealed a strong effect close to the mirror, with signal intensities up to twice as high when the mirror was used (Fig. 1). The effect was gradually lost with increased distance from the mirror. At $400-\mu \mathrm{m}$ nominal distance away from the mirror, and thus an actual distance of $500 \mu \mathrm{m}$ (see Sec. 2.3), the signal increase was still about $20 \%$. While in the upper half of the slide, signal intensity decreased with increasing distance from the top surface, in the lower half, signal intensity first leveled out and then increased when approaching the mirror. This effect was more pronounced in the blue test slide [Fig. 1(a)] than in the red test

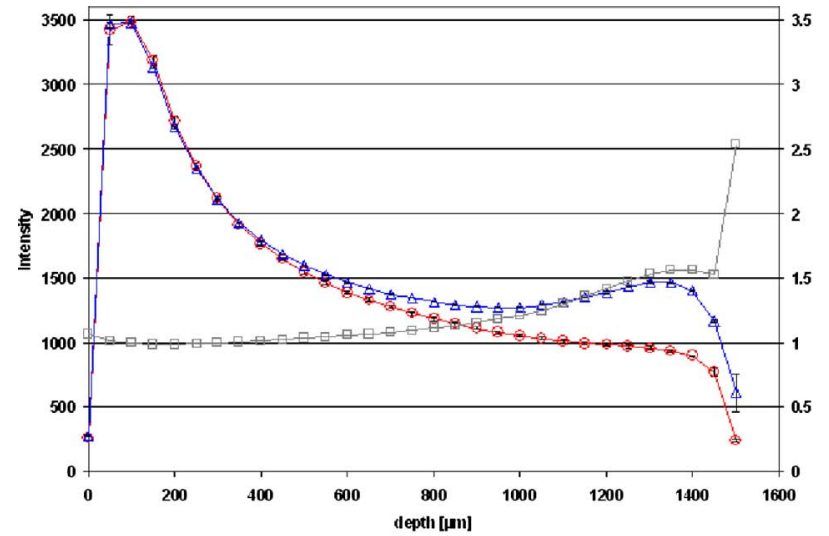

(a)

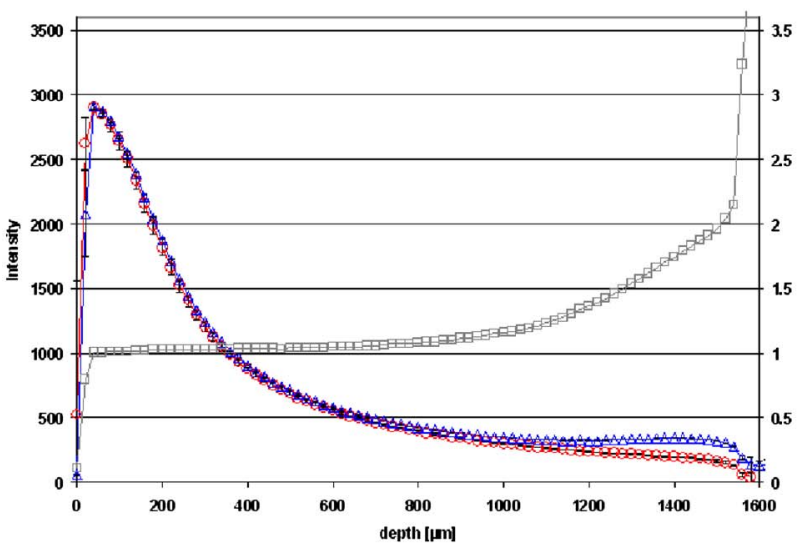

(b)

Fig. 1 Fluorescence intensity (left $y$ axis) without (red circles) and with a mirror attached underneath a test slide (blue triangles). The ratio is shown as gray squares (right $y$ axis). (a) Blue test slide, average from five $z$-stacks from different areas. (b) Red test slide, average from six $z$-stacks from different areas. Standard deviation is indicated with error bars. However, variation was so small that error bars are mostly invisible. The nominal distances from the top of the 3-D stack are given ( $x$ axis). The first measured point $(0 \mu \mathrm{m})$ is above the slide. (Color online only.)

slide [Fig. 1(b)], probably due to different absorption or scattering behavior of the slides at the respective wavelengths.

\subsection{Collagen I Matrix as an SHG-Generating Sample}

To test the effect of an underlying mirror on SHG signal intensity, we selected a specimen with a regular threedimensional distribution of harmonophores throughout a volume, a collagen I gel that was cast over a mirror and a control coverslip. Collagen is the most widely studied biological inductor of SHG signals (e.g., Refs. 6, 9, 20, and 21). Visual inspection of SHG images from several positions of the gel confirmed even collagen fiber distribution. Illumination was with $850 \mathrm{~nm}$, inducing SHG at $425 \mathrm{~nm}$. 3-D stacks with an optical thickness of over a millimeter were recorded.

Imaging paths of forward and backward signals differed in important aspects (collection through objective versus condenser, shorter path for forward detection), thus a comparison of intensities does not necessarily reflect the amount of signal 

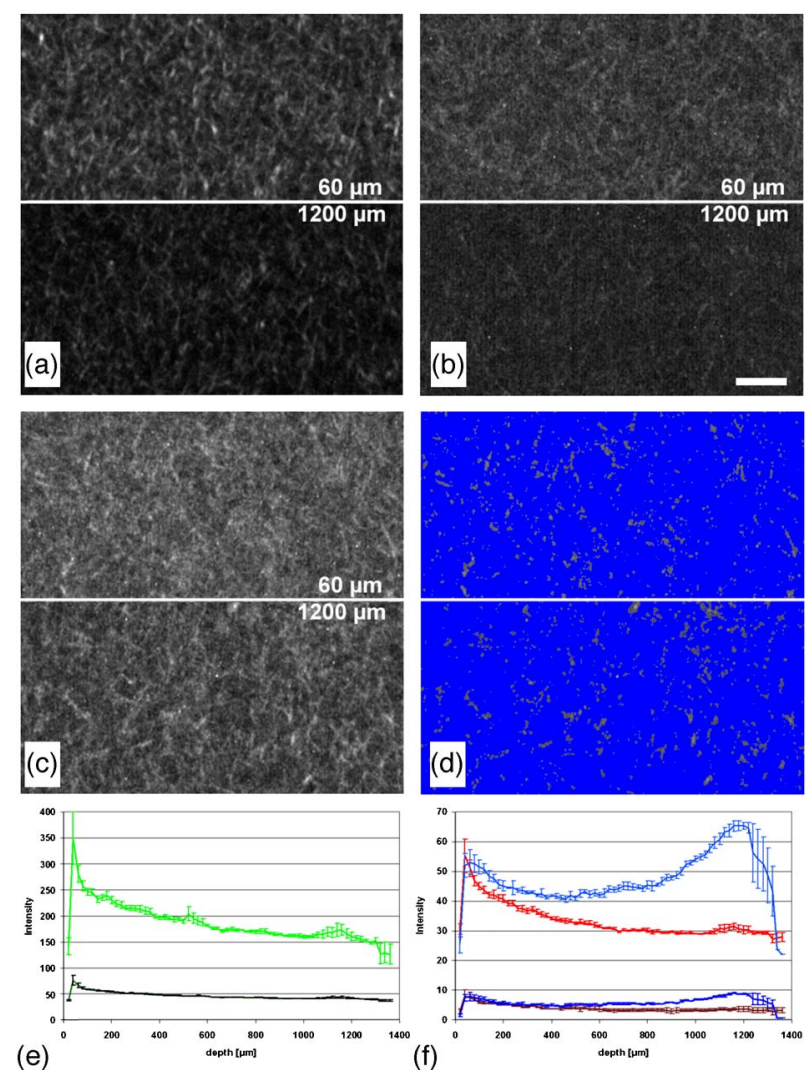

Fig. 2 SHG signals in a collagen gel. (a) Parts of optical sections from a control stack at a depth of $60 \mu \mathrm{m}$ (top) and $1200 \mu \mathrm{m}$ (bottom) recorded with the forward detector. (b) Identical sections recorded with the backward detector. (c) Sections at the same depths from a stack recorded with underlying mirror. (d) Images as in (c), with thresholding of the brightest 5\% pixels. Images in (b) and (c) were recorded with the same detector and processed identically. A different brightness adjustment had to be applied to (a) to avoid digital overexposure. Scale bar: $10 \mu \mathrm{m}$. (e) and (f) SHG signal intensity throughout the depth of a collagen gel. The average intensity of the brightest $5 \%$ pixels $[$ see $(d)]$ was measured. The average intensity of the darkest $5 \%$ (background) is given to allow an estimation of the detector baseline (dark curves). (e) Forward SHG (green); (f) backward SHG without mirror (red) and mirror-amplified SHG (blue) (Color online only.).

generated in the sample. With this limitation in mind, we found that the forward SHG signal [Fig. 2(a)] had a higher intensity and a better signal-to-noise ratio than the conventional backward signal [Fig. 2(b)], as previously described (see Sec. 1). Visual inspection showed that signals collected with the mirror [Fig. 2(c)] did not reach the signal-to-noise ratio nor the intensity of forward signals. However, mirroraugmented signals had a better signal-to-noise ratio and were brighter than conventional backward signals that were recorded with the same detector and light path and are thus directly comparable.

To confidently exclude background pixels from quantitative intensity measurements of SHG signals, we determined the brightest $5 \%$ of pixels in each section of the 3-D stack [Fig. 2(d)] and calculated their average intensity. While this procedure will not include the complete SHG signals, it allows a reproducible intensity comparison throughout image stacks. Since the fraction of the incoming light that is scat- tered in the sample increases with depth, a decrease of twophoton-induced events and thus signal intensity can be expected in a regular sample. Accordingly, we observed a steady decrease of signal intensity from the top to the bottom of 3-D stacks when recorded backward without mirror as well as with the forward detector [Figs. 2(e) and 2(f)]. However, the mirror-augmented SHG signal reached its maximal intensity close to the mirror, $1.2 \mathrm{~mm}$ away from the upper gel surface [Fig. 2(f)]. The benefit of additional light collected with the mirror thus overcompensated the decrease in signal generation caused by the reduced occurrence of the two-photon effect deep in the gel. Compared to control stacks, increased signal intensity was detectable already $1 \mathrm{~mm}$ above the mirror.

\subsection{SHG and THG in the Mouse Cremaster Muscle}

To explore whether benefits of mirror-augmented HHG signals would occur in an animal tissue, we analyzed explanted mouse cremaster muscles. This tissue contains collagen fibers and myosin. SHG by myosin ${ }^{21-24}$ and THG by striated muscle tissue ${ }^{24}$ have been described. Since the molecular structure of striated muscle is very regular, we considered muscle fibers suitable to compare signal intensities under various conditions. When spread out, explanted muscles were up to $200 \mu \mathrm{m}$ thick. To allow simultaneous recording of SHG and THG signals, we illuminated with $1270 \mathrm{~nm}$ to produce THG at $423 \mathrm{~nm}$ and SHG at $635 \mathrm{~nm}$ [Figs. 3(a)-3(d)]. Since SHG and THG signal intensities are dependent on the direction of polarization of the incoming laser beam, ${ }^{24}$ only muscle fibers that were parallel to the $x$ axis were evaluated.

Rectangular regions of interest (ROIs) were set in the brightest striated muscle fiber areas of each optical section of SHG and THG image stacks, carefully avoiding other signals such as collagen fibers. Up to four ROIs were placed in each image frame, and their mean intensity was measured.

For THG, we found that all ROIs recorded with the backward detector and without a mirror in the light path ( $n$ =141) had mean intensities below 46 (average 24, standard deviation 8.5), while all ROIs recorded with the mirror present $(n=249)$ had mean intensities above 56 (average 187, s.d. 66); the groups were thus nonoverlapping [Fig. 3(e)]. On average, mirror-augmented THG signals were 7.6 times brighter than those from control images. For SHG, all control ROIs $(n=150)$ had mean intensities below 32 (average 15, s.d. 5), while all mirrored ROIs $(n=212)$ had intensities above 44 [average 147, s.d. 77; Fig. 3(f)]. The intensity ratio between these two nonoverlapping groups was 9.6. Seven image stacks were evaluated for mirror-augmented images, and 10 for control images. Despite this, for both SHG and THG images, fewer ROIs could be defined in control images, since suitable sites with recognizable striation were often limited. We conclude that sites with low signal intensities in nonmirror control images dropped out of the evaluation and that the evaluation may thus be biased to underestimate the true intensity ratio between mirror-augmented and control image stacks. While in collagen gels, the signal intensity increased at a distance of less than $200 \mu \mathrm{m}$ toward the mirror, in the cremaster mirror-augmented signal, intensities decreased slightly [Figs. 3(e) and 3(f)], a likely consequence of stronger scattering in muscle tissue. 

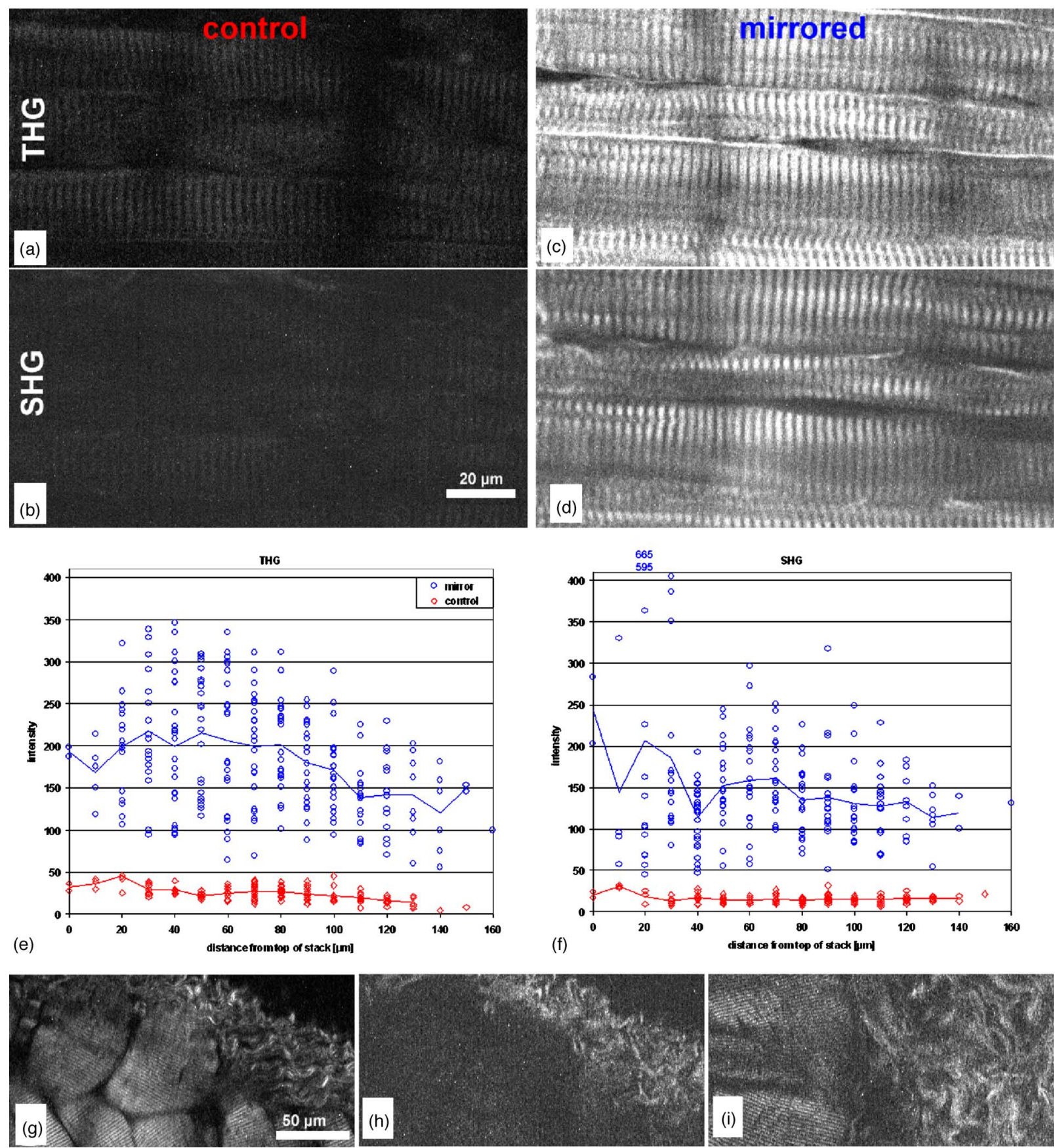

Fig. 3 Higher harmonic imaging in the mouse cremaster muscle. (a) to (f) Illumination with $1270 \mathrm{~nm}$. THG (a,c) and corresponding SHG (b,d) images recorded with the backward detector. At identical recording and image processing parameters, intensity was very low without mirror $(a, b)$ while images collected with the help of the mirror $(c, d)$ are already partially saturated. Note vertical shadows due to structures in other focal planes. (e) and (f) Quantitative analysis of signal intensity in muscle striations in THG (e) and SHG (f) image stacks. Individual intensity measurements in striated muscle are indicated by blue circles (with mirror) or red diamonds (control, no mirror). Averages are drawn as continuous lines. To allow the same scaling for both diagrams, two values in (f) had to be off chart; they are indicated numerically. (g) to (i) SHG with 860-nm illumination detected in the forward direction (g); the same optical section detected with the backward detector (h) and an area from the same muscle with mirror-augmented signal (i). (h) and (i) were processed identically. Note the absence of SHG signal from myosin in the backward-detected image at the given settings. The mirror-augmented image clearly shows muscle striation. (Color online only.)

To compare "with mirror" and "no mirror" backward signals against forward signal, we induced SHG with $860 \mathrm{~nm}$ [Figs. 3(g)-3(i)]. Due to the setup of our microscope system, this comparison was not possible with 1270 -nm illumination.
Signals from collagen fibers and myosin were brighter when recorded with the forward detector [Fig. $3(\mathrm{~g})]$ relative to control images collected with the backward detector without mirror [Fig. 3(h)], as previously published (see Sec. 4). As for the 
collagen gel, "with mirror" images [Fig. 3(i)] revealed brighter intensities and more structural detail than control backward signals.

\section{Discussion}

In the current study, we show that in multiphoton microscopy, the intensity of signals recorded with backward (epi-) detectors can be increased significantly by a simple aluminumcoated coverslip acting as mirror under the specimen. In uniformly fluorescent test specimens, we observed an increase of collected light of up to twofold for focal depths close to the mirror, comparable to a study that introduced a dichroic mirror behind the condenser (Ref. 15; see Sec. 1). HHG signals, which arise aligned to the direction of the inducing laser beam, benefited greatly from mirror-augmented detection when compared with conventional backward (epi-) detectors in all tested samples, including potato slices with starch granules (data not shown). Forward-detected HHG signals were brighter than both mirror-augmented and conventional backward-detected signals.

For SHG, while the majority of signal is usually generated in the forward direction, ${ }^{8}$ the actual amount of conventional backward-detected signal depends on the optical properties of the light paths to the detectors and on the structure of the sample. ${ }^{25-27}$ For example, we noted a much higher increase in intensity in mirror-augmented signals from muscle tissue than in a collagen gel. Accordingly, published forward to backward SHG signal intensity ratios of 478:1, 25:1 (Ref. 25), 20:1 (Ref. 10), 10:1 (Ref. 28), 5:1 (Ref. 13), 4:1 (Ref. 25), and 1:1 (Refs. 20 and 29) were described with various specimens and optical systems. Here, we show an up-to-ninefold intensity increase in mirror-augmented SHG signal compared to conventional backward signal. Although most likely only a part of the forward signal will be directed by the mirror to the backward detector, this increase fits with published forwardto-backward ratios.

THG microscopy has been applied less frequently. It appears that backward-detected THG signal from unstained biological specimens stems exclusively from nonballistic, multiply scattered photons originally generated in the forward detection. ${ }^{30}$ Theoretical considerations also suggest that the angular width of the forward-generated signal is narrower for THG than for SHG, ${ }^{8}$ which should lead to a better yield of reflected, collected photons for THG than for SHG in our setup with a mirror placed on the far side of the sample. However, we observed a higher increase for mirroraugmented SHG signals than for mirror-augmented THG signals, compared to normal backward-collected signals. We consider it likely that this is due to the stronger scattering of the shorter wavelength THG signal.

Our results outline potential fields of application for mirror-augmented signal detection. Obviously, it is restricted to at least partially transparent specimens, a limitation shared with forward detection. However, sometimes forward detection is technically not possible, e.g., due to spatial restrictions induced by animal anatomy. Improvement of SHG and THG signal strength in such situations is a strong candidate for application of signal reflection with a simple mirror.
An example is intravital microscopy of the cremaster muscle of the mouse. The cremaster covers the testis. With its thickness of about $200 \mu \mathrm{m}$ and the possibility to exteriorize the muscle from the body as a thin sheet of intact tissue while blood perfusion remains intact, the cremaster is a frequently used model system in intravital microscopy (e.g., Refs. 31-33). This particular muscle is thin enough to allow forward collection of SHG and THG signals, as we have shown in this study. However, for intravital microscopy, it is not possible to position the mouse such that the cremaster is accessible for both immersion objective from above and condenser from below. Putting instead a mirror under the cremaster to collect higher signal intensities is a feasible alternative. Other examples for mouse tissues with similar spatial restrictions are the ear and the mesentery. Mirror-augmented detection may also be applicable in clinical investigations of collagen or myosin organization with SHG or of nerves or other structures with THG. While the introduction of relaying optics behind the structure of interest may be impossible, the minimally invasive introduction of a small mirror may significantly increase signal strength and thus reliability of results.

From a practical point of view, mirror-augmented detection is also able to improve collection of HHG signals on microscope systems that lack forward detectors. Basic systems designed for two-photon fluorescence do not feature such detectors. Here, mirror-augmented detection will allow, for example, increased signal-to-noise ratios for SHG induced by collagen and thus ease orientation: many animal tissues contain substantial amounts of collagen fibers, especially so at their surface, which outline the structural organization of the organ.

Apart from sample transparency, the only condition for the collection of mirror-reflected signals is the restriction of signal generation to a single spot. This condition is fulfilled also in other types of nonlinear microscopy. Therefore, mirroraugmented signal collection should also be applicable to, e.g., coherent anti-Stokes Raman scattering (CARS) microscopy.

\section{Acknowledgments}

We thank Jerome Mutterer, Strasbourg, for providing an ImageJ macro (see Sec. 2.4). This work was supported by the BioImaging Network of Ludwig-Maximilians-Universität München and the Excellence Initiative of Deutsche Forschungsgemeinschaft and Bundesministerium für Bildung und Forschung.

\section{References}

1. V. Centonze and J. B. Pawley, "Tutorial on practical confocal microscopy and use of the confocal test specimen," in Handbook of Biological Confocal Microscopy, J. B. Pawley, Ed., pp. 627-649, Springer Science and Business Media, New York (2006).

2. W. Denk, D. W. Piston, and W. W. Webb, "Multi-photon molecular excitation in laser-scanning microscopy," in Handbook of Biological Confocal Microscopy, J. B. Pawley, Ed., pp. 535-549, Springer Science and Business Media, New York (2006).

3. S. Hell and E. H. K. Stelzer, "Fundamental improvement of resolution with a 4pi-confocal fluorescence microscope using 2-photon excitation," Opt. Commun. 93(5-6), 277-282 (1992).

4. S. W. Hell, "Far-field optical nanoscopy," Science 316(5828), 1153 1158 (2007).

5. S. Lindek and E. H. Stelzer, "Resolution improvement by nonconfo- 
cal theta microscopy," Opt. Lett. 24(21), 1505-1507 (1999).

6. P. Friedl, K. Wolf, U. H. von Andrian, and G. Harms, "Biological second and third harmonic generation microscopy," Curr. Protoc. Cell Biol. 4, 4.15 (2007).

7. P.-C. Cheng and C. K. Sun, "Nonlinear (harmonic generation) optical microscopy," in Handbook of Biological Confocal Microscopy, J. B. Pawley, Ed., pp. 703-721, Springer Science and Business Media, New York (2006).

8. L. Moreaux, O. Sandre, S. Charpak, M. Blanchard-Desce, and J." Mertz, "Coherent scattering in multi-harmonic light microscopy," Biophys. J. 80(3), 1568-1574 (2001).

9. G. Cox and E. Kable, "Second-harmonic imaging of collagen," Methods Mol. Biol. 319, 15-35 (2006).

10. G. Cox, E. Kable, A. Jones, I. Fraser, F. Manconi, and M. D. Gorrell, "3-dimensional imaging of collagen using second harmonic generation," J. Struct. Biol. 141(1), 53-62 (2003).

11. P. J. Campagnola and L. M. Loew, "Second-harmonic imaging microscopy for visualizing biomolecular arrays in cells, tissues and organisms," Nat. Biotechnol. 21(11), 1356-1360 (2003)

12. C. K. Sun, S. W. Chu, S. Y. Chen, T. H. Tsai, T. M. Liu, C. Y. Lin, and H. J. Tsai, "Higher harmonic generation microscopy for developmental biology," J. Struct. Biol. 147(1), 19-30 (2004).

13. N. Moreno, J. A. Feijo, and G. Cox, "Implementation and evaluation of a detector for forward propagated second harmonic signals," $\mathrm{Mi}$ cron 35(8), 721-724 (2004).

14. R. Gauderon, P. B. Lukins, and C. J. Sheppard, "Three-dimensional second-harmonic generation imaging with femtosecond laser pulses," Opt. Lett. 23(15), 1209-1211 (1998).

15. D. L. Wokosin, J. M. Squirrell, K. W. Eliceiri, and J. G. White, "Optical workstation with concurrent, independent multiphoton imaging and experimental laser microbeam capabilities," Rev. Sci. Instrum. 74(1), 193-201 (2003).

16. C. A. Combs, A. V. Smirnov, J. D. Riley, A. H. Gandjbakhche, J. R. Knutson, and R. S. Balaban, "Optimization of multiphoton excitation microscopy by total emission detection using a parabolic light reflector," J. Microsc. 228(Pt 3), 330-337 (2007).

17. T. R. Mempel, C. Moser, J. Hutter, W. M. Kuebler, and F. Krombach, "Visualization of leukocyte transendothelial and interstitial migration using reflected light oblique transillumination in intravital video microscopy," J. Vasc. Res. 40(5), 435-441 (2003).

18. A. Egner and S. W. Hell, "Aberrations in confocal and multi-photon fluorescence microscopy induced by refractive index mismatch," in Handbook of Biological Confocal Microscopy, J. B. Pawley, Ed., pp. 404-413, Springer Science and Business Media, New York (2006).

19. P. Reichardt, F. Gunzer, and M. Gunzer, "Analyzing the physicodynamics of immune cells in a three-dimensional collagen matrix," Methods Mol. Biol. 380, 253-269 (2007).

20. M. Han, G. Giese, and J. Bille, "Second harmonic generation imaging of collagen fibrils in cornea and sclera," Opt. Express 13(15), 57915797 (2005)
21. P. J. Campagnola, A. C. Millard, M. Terasaki, P. E. Hoppe, C. J. Malone, and W. A. Mohler, "Three-dimensional high-resolution second-harmonic generation imaging of endogenous structural proteins in biological tissues," Biophys. J. 82(1 Pt 1), 493-508 (2002).

22. M. Both, M. Vogel, O. Friedrich, F. von Wegner, T. Kunsting, R. H. Fink, and D. Uttenweiler, "Second harmonic imaging of intrinsic signals in muscle fibers in situ," J. Biomed. Opt. 9(5), 882-892 (2004).

23. S. V. Plotnikov, A. C. Millard, P. J. Campagnola, and W. A. Mohler, "Characterization of the myosin-based source for second-harmonic generation from muscle sarcomeres," Biophys. J. 90(2), 693-703 (2006).

24. S. W. Chu, S. Y. Chen, G. W. Chern, T. H. Tsai, Y. C. Chen, B. L. Lin, and C. K. Sun, "Studies of chi(2)/chi(3) tensors in submicronscaled bio-tissues by polarization harmonics optical microscopy," Biophys. J. 86(6), 3914-3922 (2004).

25. F. Légaré, C. Pfeffer, and B. R. Olsen, "The role of backscattering in SHG tissue imaging," Biophys. J. 93(4), 1312-1320 (2007).

26. W. R. Zipfel, R. M. Williams, R. Christie, A. Y. Nikitin, B. T. Hyman, and W. W. Webb, "Live tissue intrinsic emission microscopy using multiphoton-excited native fluorescence and second harmonic generation," Proc. Natl. Acad. Sci. U.S.A. 100(12), 7075-7080 (2003).

27. S. W. Chu, S. P. Tai, T. M. Liu, C. K. Sun, and C. H. Lin, "Selective imaging in second-harmonic-generation microscopy with anisotropic radiation," J. Biomed. Opt. 14(1), 010504 (2009).

28. D. A. Dombeck, K. A. Kasischke, H. D. Vishwasrao, M. Ingelsson, B. T. Hyman, and W. W. Webb, "Uniform polarity microtubule assemblies imaged in native brain tissue by second-harmonic generation microscopy," Proc. Natl. Acad. Sci. U.S.A. 100(12), 7081-7086 (2003).

29. R. M. Williams, W. R. Zipfel, and W. W. Webb, "Interpreting secondharmonic generation images of collagen I fibrils," Biophys. J. 88(2), 1377-1386 (2005)

30. D. Debarre, N. Olivier, and E. Beaurepaire, "Signal epidetection in third-harmonic generation microscopy of turbid media," Opt. Express 15(14), 8913-8924 (2007).

31. S. Baez, "An open cremaster muscle preparation for the study of blood vessels by in vivo microscopy," Microvasc. Res. 5(3), 384-394 (1973).

32. A. G. Khandoga, A. Khandoga, C. A. Reichel, P. Bihari, M. Rehberg, and F. Krombach, "In vivo imaging and quantitative analysis of leukocyte directional migration and polarization in inflamed tissue," PLOS ONE 4(3), e4693 (2009).

33. C. Sumen, T. R. Mempel, I. B. Mazo, and U. H. von Andrian, "Intravital microscopy: visualizing immunity in context," Immunity 21(3), 315-329 (2004)

34. M. Müller and A. Zumbusch, "Coherent anti-Stokes Raman scattering microscopy," ChemPhysChem 8(15), 2156-2170 (2007). 